\title{
Fabrication and characterization of drug-loaded nano-hydroxyapatite/polyamide 66 scaffolds modified with carbon nanotubes and silk fibroin
}

This article was published in the following Dove Press journal:

International Journal of Nanomedicine

18 November 2016

Number of times this article has been viewed

\author{
Meng-Zhu Yao' \\ Ming-Yi Huang-Fu' \\ Hui-Na Liu' \\ Xia-Rong Wang' \\ Xiaoxia Sheng ${ }^{2}$ \\ Jian-Qing Gao'
}

'Institute of Pharmaceutics, College of Pharmaceutical Sciences, Zhejiang University, ${ }^{2}$ Hangzhou SoliPharma

Co., Ltd, Hangzhou, Zhejiang, People's Republic of China
Correspondence: Jian-Qing Gao Institute of Pharmaceutics, College of Pharmaceutical Sciences, Zhejiang University, 866 Yuhangtang Road, Hangzhou 310058, Zhejiang, People's Republic of China

$\mathrm{Tel}+8657188208436$

Fax +86 57I 88208436

Email gaojianqing@zju.edu

\begin{abstract}
Nano-hydroxyapatite/polyamide 66 (nHA/PA66) porous scaffolds were fabricated by a phase inversion method. Carbon nanotubes (CNTs) and silk fibroin (SF) were used to modify the surface of the nHA/PA66 scaffolds by freeze-drying and cross-linking. Dexamethasone was absorbed to the CNTs to promote the osteogenic differentiation of bone mesenchymal stem cells (BMSCs). The cell viability of BMSCs was investigated by changing the concentration of the CNT dispersion, and the most biocompatible scaffold was selected. In addition, the morphology and mechanical property of the scaffolds were investigated. The results showed that the nHA/PA66 scaffolds modified with CNTs and SF met the requirements of bone tissue engineering scaffolds. The dexamethasone-loaded CNT/SF-nHA/PA66 composite scaffold promoted the osteogenic differentiation of BMSCs, and the drug-loaded scaffolds are expected to function as effective bone tissue engineering scaffolds.
\end{abstract}

Keywords: BMSCs, tissue engineering, porous scaffold, carbon nanotubes, silk fibroin, surface modification, dexamethasone

\section{Introduction}

Bone defects caused by infections, congenital malformations, trauma, or bone tumors are commonly seen in the clinic. ${ }^{1}$ Traditional treatments include autogenous bone graft, allograft bone transplantation, and artificial bone material transplantation. However, these three traditional treatments have some limitations. ${ }^{2}$ In 1995 , Crane et al proposed the concept of bone tissue engineering, which attracted the attention of scholars. ${ }^{3}$ With the development of life, material, and engineering sciences, bone tissue engineering research has advanced in recent years ${ }^{4}$ and has been currently the primary approach to bone defect repair. The tissue scaffold is the key factor for bone tissue engineering. ${ }^{5}$ It promotes the adhesion and growth of seed cells and new cells. ${ }^{6,7}$

The materials used for bone tissue engineering scaffolds are divided into three categories: biological derivative materials, inorganic materials, and polymer materials. ${ }^{8}$ However, it is often difficult to meet the requirements of an ideal bone tissue scaffold by using only a single material. The mechanical properties and electrical performance of biological derivative materials are not ideal. The cell compatibility and mechanical performance of synthetic polymer materials are poor. ${ }^{9}$ Inorganic materials are usually very brittle. Natural polymer materials have undesirable batch-to-batch consistency and mechanical properties. Due to advantages and disadvantages of single-type materials mentioned above, composite scaffolds containing two or more different types of components have often been considered to exhibit improved characteristics by protruding 
the strengths while evading the weaknesses of the individual components. ${ }^{10,11}$ For example, the stability of mechanically weaker polymers with excellent biocompatibility, such as chitosan, could be reinforced by incorporating mechanically strong composites.

Nano-hydroxyapatite/polyamide 66 (nHA/PA66) has been widely used as an orthopedics transplantation material for bone cysts, osteofibrous dysplasia, aseptic bone necrosis, and bone defect repair. The crystal structure and composition of nHA crystals are similar to those of natural bone; PA66 is structurally similar to collagen. Studies have shown that nHA/PA66 porous scaffolds have good blood compatibility, tissue compatibility, and bone biomechanical properties as well as good toughness for use in hard tissue repair. ${ }^{12,13}$

Based on the positive results obtained with two-phase composites, multiphase porous composite scaffold materials and antibacterial, osteogenesis, or angiogenesis functional scaffolds have attracted widespread attention. ${ }^{14-16}$ Carbon nanotubes (CNTs), which have excellent mechanical properties, are one type of material used for building ideal composite bone tissue engineering scaffolds, and they have been widely used to build composite scaffolds. ${ }^{17-19}$ Silk fibroin (SF) is a natural polymer extracted from silk fibrous protein. SF has good mechanical properties, flexibility and tensile strength, ${ }^{20}$ and permeability as well as slow-releasing potential. ${ }^{21}$ It has been widely recognized as a natural biodegradable biological material. In addition, SF contains acidic proteins whose side chains are rich in carboxylic acid groups that can induce the mineralization of calcium phosphate, which plays a very important role in bone mineralization. ${ }^{22}$ These qualities make SF a popular choice for bone tissue engineering in recent years. ${ }^{23}$

In this study, nHA/PA66 was surface modified with SF and CNTs in order to improve the properties of the nHA/ PA66 scaffold. In addition, CNTs were used to load dexamethasone (DEX) to promote the differentiation of bone mesenchymal stem cells (BMSCs).

\section{Materials and methods Materials}

Multi walled carbon nanotubes modified by carboxyl (MWCNT-COOH; Cat No TNSMC5) was purchased from Chengdu Organic Chemicals, Chinese Academy of Sciences (Chengdu, People's Republic of China). nHA/PA66 porous bone ( $4 \mathrm{~mm}$ width $\times 4 \mathrm{~mm}$ length $\times 40 \mathrm{~mm}$ height) was provided by Guona Science and Technology Co., Ltd (Sichuan, People's Republic of China). The weight ratio of nHA to PA66 was 0.2-1.5:100. DEX (purity $>98 \%$ ) was purchased from Sigma-Aldrich Co. (St Louis, MO, USA). Dulbecco's Modified Eagle's Medium (DMEM) was provided by Hyclone (Logan, UT, USA), and fetal bovine serum was provided by Thermo Fisher Scientific (Waltham, MA, USA). Sprague Dawley (SD) rat mesenchymal stem cell osteogenic differentiation medium was provided by Cyagen Biosciences Inc. (Santa Clara, CA, USA). Water-soluble tetrazolium salt assay and alkaline phosphatase (ALP) activity detection kits were purchased from Beyotime Biotechnology Company (Jiangsu, People's Republic of China). Live/dead cell image kit was provided by Thermo Fisher Scientific. $N$-(3-dimethyl amino propyl)- $N$ '-ethylcarbodiimide hydrochloride (EDC) was purchased from Sigma-Aldrich Co. $N$-hydroxysuccinimide (NHS) was purchased from Aladdin Co. (Shanghai, People's Republic of China). Methyl alcohol and anhydrous ethanol were purchased from Tianjin Shield (Tianjin, People's Republic of China). Acetonitrile was obtained from Merck \& Co., Inc. (Kenilworth, NJ, USA). Digital multimeter MY68 was from Mastech (Guangzhou, People's Republic of China).

\section{Animals}

SD rats (male, 3 weeks old) were purchased from Shanghai SLAC Laboratory Animal Co. Ltd. (Shanghai, People's Republic of China). All animal experiments were performed with the approval of the Animal Experimental Ethics Committee of Zhejiang University. All animals were handled in accordance with the National Institutes of Health Guidelines for the Care and Use of Laboratory Animals.

\section{Methods}

\section{Preparation of porous three-dimensional scaffolds}

The CNT/SF-nHA/PA66 scaffold was prepared as shown in Figure 1. The CNT/SF mixture was prepared as follows. SF and CNT were dispersed in distilled deionized water to produce $0.2 \mathrm{wt} \% \mathrm{SF}$ solution and $5 \mathrm{mg} / \mathrm{mL}$ CNT dispersion, respectively. To each SF solution, a given amount of the aforementioned CNT dispersion was added to produce CNT/SF mixture with various CNT concentrations $(0.2,0.5$, and $1 \mathrm{mg} / \mathrm{mL})$. The nHA/PA66 scaffolds $(1 \mathrm{~cm}$ width $\times 1 \mathrm{~cm}$ length $\times 15 \mathrm{~cm}$ height) were dipped in SF or CNT/SF mixture and kept at room temperature for 24 hours. After infiltration, all the scaffolds were frozen at $-80^{\circ} \mathrm{C}$ for 4 hours and then lyophilized for 48 hours. Then, these scaffolds were crosslinked using $50 \mathrm{mmol} / \mathrm{L}$ EDC and $18 \mathrm{mmol} / \mathrm{L}$ NHS. The EDC/NHS solution was prepared by dissolving $179.69 \mathrm{mg}$ EDC and $41.432 \mathrm{mg}$ NHS in $20 \mathrm{~mL}$ of $90 \%$ ethyl alcohol. To each $20 \mathrm{~mL}$ of EDC/NHS solution, $200 \mathrm{mg}$ of dry scaffold was 


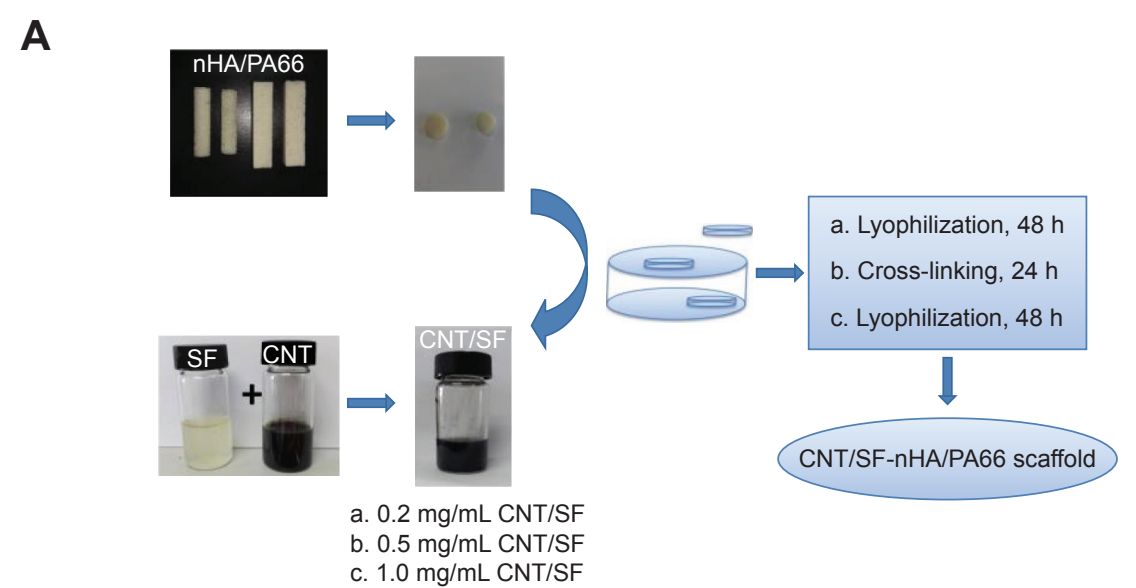

B

Figure I Preparation of scaffolds.

Notes: (A) Preparation of CNT/SF-nHA/PA66 scaffold. (B) Preparation of DEX-loaded CNT/SF-nHA/PA66 scaffold.

Abbreviations: CNT, carbon nanotube; DEX, dexamethasone; EDC, $\mathrm{N}$-(3-dimethyl amino propyl)- $\mathrm{N}^{\prime}$-ethylcarbodiimide hydrochloride; nHA, nano-hydroxyapatite; NHS, N-hydroxysuccinimide; PA66, polyamide 66; SF, silk fibroin.

added and the scaffolds were cross-linked at room temperature for 90 minutes. The cross-linked scaffolds were repeatedly washed with distilled water and lyophilized again.

\section{Scaffold characterization}

The morphologies of the nHA/PA66, SF-nHA/PA66, and CNT/SF-nHA/PA66 scaffolds were gold-sprayed and viewed using the field emission scanning electron microscope (SEM) (SU8010; Hitachi Ltd., Tokyo, Japan).

Fourier transform infrared spectra of the nHA/PA66, SF-nHA/PA66, and CNT/SF-nHA/PA66 scaffolds were recorded on an infrared spectrometer $(1.0 \mathrm{mg}$ sample in $100 \mathrm{mg}$ of $\mathrm{KBr}$ powder) in the transmittance mode. The samples were prepared by cutting each scaffold into very thin sheets and grinding each sheet into powder.

The crystallinity of nHA/PA66 and CNT/SF-nHA/PA66 was investigated with an X-ray powder diffractometer (Shimadzu, Kyoto, Japan) using $\mathrm{CuK} \alpha(\lambda=1.54 \AA)$ at $40 \mathrm{kV}$ and $35 \mathrm{~mA}$. The crystallinity phases of the samples were determined using diffraction angles from $10^{\circ}$ to $80^{\circ}$.

The mechanical parameters of the nHA/PA66 and CNT/ SF-nHA/PA66 scaffolds were measured using a mechanical testing machine (AG-1, Shimadzu) with a crosshead speed of
$0.5 \mathrm{~mm} / \mathrm{min}$. Cube-shaped scaffolds ( $1 \mathrm{~cm}$ width, $1 \mathrm{~cm}$ length, and $15 \mathrm{~cm}$ height) were used for the compression tests.

The porosity of the nHA/PA66 and CNT/SF-nHA/PA66 scaffolds was determined using a mercury porosimeter (AutoPore IV 9510; Micromeritics Instrument Corporation, Norcross, GA, USA). All the necessary calculations were performed using the software Micromeritics Analyzer (Micromeritics Instrument Corporation).

The nHA/PA66 and CNT/SF-nHA/PA66 scaffolds were immersed in distilled water at room temperature for 24 hours to measure their swelling properties. After removing the excess water, the wet weight of the scaffolds $\left(\mathrm{W}_{1}\right)$ was measured. Samples were then dried in an oven at $60^{\circ} \mathrm{C}$ under vacuum overnight, and the dry weight of the scaffolds $\left(\mathrm{W}_{2}\right)$ was measured. The water absorption percentage of the scaffolds was calculated using the following equation:

$$
\text { Water absorption } \%=\left(\mathrm{W}_{1}-\mathrm{W}_{2}\right) / \mathrm{W}_{1} \times 100 \%
$$

The conductivity of each CNT/SF-nHA/PA66 scaffold was measured using the method described earlier. ${ }^{24}$ Sample surfaces were coated with silver paste to reduce contact resistance between the sample and the electrodes. Two metal 
electrodes were connected to a digital multimeter (MY68, Mastech). Hydrated samples, which were $5 \times 5 \times 2 \mathrm{~mm}^{3}$ rectangle plates, were tightly clamped between the two electrodes for the measurement. The resistance (R) measurement was carried out ten times on each sample. $\mathrm{R}$ can be obtained directly from the multimeter, and thus, the electrical conductivity $\sigma$ can be calculated using the Pouillet's law:

$$
\sigma=\frac{L}{R \times A}
$$

where $\mathrm{L}$ is the thickness and $\mathrm{A}$ is the surface area of samples.

\section{Degradation properties}

Biodegradation of CNT/SF-nHA/PA66 scaffolds was evaluated in simulated body fluid (SBF) at $37^{\circ} \mathrm{C}$. The SBF was prepared as described by Kokubo and Takadama. ${ }^{25}$ After the CNT/SF-nHA/PA66 scaffolds were soaked in SBF for 1, 3, $5,12,15,20,24$, and 30 days, the scaffolds were removed and washed with deionized water. Then, the scaffolds were dried at $37^{\circ} \mathrm{C}$ in a constant-temperature oven and weighed, and change in the $\mathrm{pH}$ value of the SBF was measured. The in vitro biodegradation rate was calculated as follows:

$$
\text { Degradation rate }(\%)=(\mathrm{D} 1-\mathrm{D} 2) / \mathrm{D} 1 \times 100 \%
$$

D1 represents the initial weight of the CNT/SF-nHA/PA66 scaffold and D2 represents the final weight of the CNT/SFnHA/PA66 scaffold after exposure to the degradation solution. Data were expressed as mean \pm standard deviation $(n=3)$.

\section{BMSC isolation, purification, and culture}

Rat bone marrow-derived mesenchymal stem cells were isolated and cultured by the adherence method. The bone marrow of the femora of 3-week-old SD rats was harvested using a previously reported protocol. ${ }^{26}$ The details can be found in Supplementary materials.

\section{Cell viability}

The different scaffolds modified by CNT/SF solution with various CNT concentrations $(0.2,0.5$, and $1 \mathrm{mg} / \mathrm{mL})$ were evaluated according to the cell viability of BMSCs cultured on the scaffolds. First, the nHA/PA66, SF-nHA/PA66, $0.2 \mathrm{mg} / \mathrm{mL}$ CNT/SF-nHA/PA66, $0.5 \mathrm{mg} / \mathrm{mL}$ CNT/SF-nHA/ PA66, and $1 \mathrm{mg} / \mathrm{mL}$ CNT/SF-nHA/PA66 scaffolds were wetted in low-glucose DMEM (GL-DMEM) complete medium for 4 hours to moisten the scaffolds, after which they were transferred to $24-$ well culture plates. Then, the third passage of BMSCs $\left(1 \times 10^{5}\right.$ cells $\left./ 10 \mu \mathrm{L}\right)$ was seeded onto the top of the prewetted scaffolds. The seeded scaffolds were incubated at $37^{\circ} \mathrm{C}$ in a humidified atmosphere with $5 \% \mathrm{CO}_{2}$ for 3 hours to allow cell attachment. Then, $1 \mathrm{~mL}$ of GL-DMEM complete medium was added to each well. The medium was changed every 3 days. The cell scaffolds were then cultured for 7 days. Cells were washed three times in phosphate-buffered saline (PBS). Then, the cells were visualized using confocal laser scanning microscopy (IX81-FV1000; Olympus Corporation, Tokyo, Japan). The emission wavelength and the excitation wavelength of the green fluorescence observed were at 488 and $518 \mathrm{~nm}$, respectively; the emission wavelength and the excitation wavelength of the red fluorescence observed were 488 and $615 \mathrm{~nm}$, respectively. Finally, MetaMorph software (Molecular Devices, Sunnyvale, CA, USA) was used to analyze the amount of red fluorescence ( $\sum$ Scaffold area) and green fluorescence ( $\Sigma$ Cell area) and calculate the cell viability per unit volume. The cell viability of BMSCs on the scaffold was calculated using the following equation:

$$
\text { Cell viability } \%=\frac{\sum \text { Cell area }}{\sum \text { Scaffold area }} \times 100 \%
$$

\section{Cell attachment}

SEM was used to investigate the morphology of BMSCs grown on the different scaffolds. The samples were prepared by washing them twice with $\mathrm{PBS}$ ( $\mathrm{pH} 7.4$ ); they were then fixed with $4 \%$ paraformaldehyde in $\mathrm{PBS}$ at $4^{\circ} \mathrm{C}$ for 20 minutes. Subsequently, these samples were fixed by immersing in $2.5 \%$ osmic acid solution for 24 hours at $4{ }^{\circ} \mathrm{C}$. The samples were then subjected to acetone dehydration with critical point drying, sputter-coated with gold, and viewed using an SEM at an accelerating voltage of $15 \mathrm{kV}$.

\section{Drug release test}

Drug release was investigated by placing the DEX-loaded CNT/SF-nHA/PA66 scaffold into a dialysis bag (3,000 MW) containing different release media: PBS ( $\mathrm{pH} 7.4)$ and $0.5 \%$ sodium dodecyl sulfate (SDS) in PBS. The DEX-loaded CNT/SF-nHA/PA66 scaffold in the dialysis bag was placed in a centrifuge tube containing $50 \mathrm{~mL}$ of the release medium. The centrifuge tube was placed in shaker incubator at $37^{\circ} \mathrm{C}$ and $50 \mathrm{rpm}$. At 15 minutes, 30 minutes, 1 hour, 2 hours, 3 hours, 6 hours, 8 hours, 18 hours, 24 hours, 2 days, 4 days, and 6 days, $0.5 \mathrm{~mL}$ of the release medium (sample) was withdrawn from the centrifuge tube and replaced with $0.5 \mathrm{~mL}$ of fresh release medium. The samples were analyzed 
by high-performance liquid chromatography (Agilent 1200; Agilent Technologies, Santa Clara, CA, USA).

\section{ALP activity assay}

The different drug-loaded CNT/SF-nHA/PA66 scaffolds with various concentrations of $\operatorname{DEX}(0.1,1$, and $5 \mathrm{mg} / \mathrm{mL})$ were evaluated regarding ALP activity of BMSCs on the scaffold. First, the nHA/PA66, $0.1 \mathrm{mg} / \mathrm{mL}$ DEX-CNT/SF-nHA/PA66, $1 \mathrm{mg} / \mathrm{mL}$ DEX-CNT/SF-nHA/PA66, and $5 \mathrm{mg} / \mathrm{mL}$ DEXCNT/SF-nHA/PA66 scaffolds were wetted in GL-DMEM complete medium for 4 hours to moisten the scaffold, after which they were transferred to $24-w e l l$ culture plates. Then, the third passage of BMSCs $\left(1 \times 10^{6}\right.$ cells $\left./ 10 \mu \mathrm{L}\right)$ was seeded onto the top of the prewetted scaffolds. The seeded scaffolds were incubated at $37^{\circ} \mathrm{C}$ in a humidified atmosphere with $5 \% \mathrm{CO}_{2}$ for 3 hours to allow cell attachment. Then, $1 \mathrm{~mL}$ of GL-DMEM complete medium was added to each well. The medium was changed every 3 days. The culture was aspirated and washed with PBS three times, and then $0.1 \%$ Triton- $100(500 \mu \mathrm{L})$ was added to each well followed by incubation overnight at $4^{\circ} \mathrm{C}$. The BMSCs on the scaffold were completely smashed by repeat blows and then centrifuged at 3,000 rpm for 15 minutes. The supernatant was used to determine the ALP activity with para-nitrophenyl phosphate as the substrate; the supernatant was incubated at $37^{\circ} \mathrm{C}$ for 20 minutes and the reaction terminating solution (100 $\mu \mathrm{L}$ ) was subsequently added into each well. Absorbance at
$405 \mathrm{~nm}$ was measured by a spectrophotometer with a 96-well microplate reader (Biotek, Winooski, VT, USA).

\section{Results}

\section{Morphological analysis}

Studies have reported that large holes can provide space for new bone tissue growth and small holes can provide nutrition for the tissues by capillary action. The minimum aperture size that satisfies the essential requirements for bone tissue regeneration is $\sim 100-150 \mu \mathrm{m} .{ }^{27}$ The SEM images presented in Figure 2 show the morphological characteristics of the $\mathrm{nHA} /$ PA66, SF-nHA/PA66, and CNT/SF-nHA/PA66 scaffolds. The average diameters of the scaffolds were $\sim 500 \mu \mathrm{m}$. No significant differences were observed between the diameters of the nHA/PA66 and modified nHA/PA66 scaffolds. Moreover, the SEM images of the SF-nHA/PA66 scaffold showed a protein-like structure (Figure $2 \mathrm{E}$ ). In addition, the CNTs could be observed in the SEM images of the CNT/SF-nHA/ PA66 scaffold (Figure 2F and G).

\section{Structural analysis}

The structural changes in the nHA/PA66 scaffold after surface modification were determined by Fourier transform infrared spectra. Figure $3 \mathrm{~A}$ shows the amine peaks at $1,641 \mathrm{~cm}^{-1}, 1,539 \mathrm{~cm}^{-1}$, and $3,302 \mathrm{~cm}^{-1}$; an $\mathrm{O}-\mathrm{C}=\mathrm{C}-$ stretching vibration absorption peak at $1,641 \mathrm{~cm}^{-1}$; an $\mathrm{N}-\mathrm{H}$ bending vibration peak at $1,539 \mathrm{~cm}^{-1}$; and $\mathrm{PO}_{4}^{3-}$
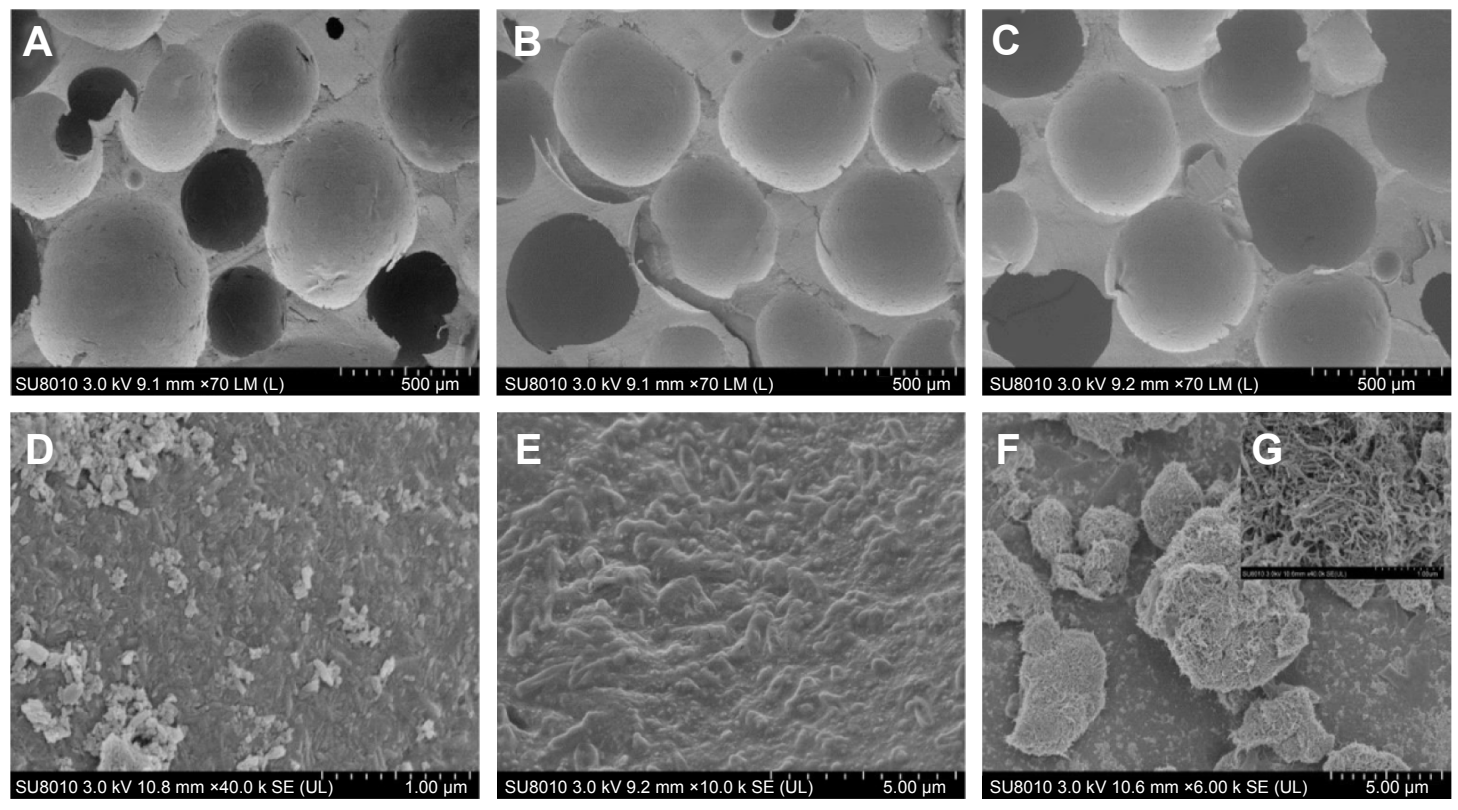

Figure 2 SEM images of (A, D) nHA/PA66, (B, E) SF-nHA/PA66, (C, F, G) $0.2 \mathrm{mg} / \mathrm{mL}$ CNT/SF-nHA/PA66.

Notes: The average diameters of the scaffolds were $\sim 500 \mu \mathrm{m}$. SF-nHA/PA66 scaffold showed a protein-like structure (E). In addition, the CNTs could be observed in the SEM images of the CNT/SF-nHA/PA66 scaffold (F and $\mathbf{G})$. (A-C) Scale bar $=500 \mu \mathrm{m}$, (D) scale bar $=1 \mu \mathrm{m},(\mathbf{E}-\mathbf{F})$ scale bar $=5 \mu \mathrm{m}$, and $(\mathbf{G})$ scale bar $=1 \mu \mathrm{m}$.

Abbreviations: CNT, carbon nanotube; nHA, nano-hydroxyapatite; PA66, polyamide 66; SEM, scanning electron microscopy; SF, silk fibroin. 
A
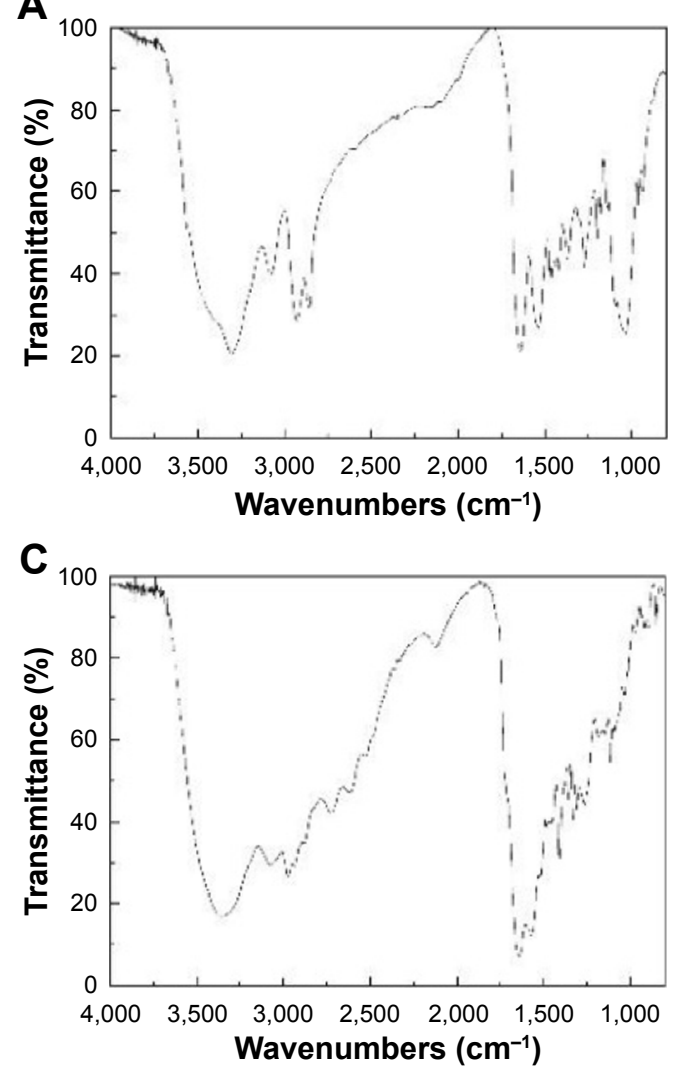

B

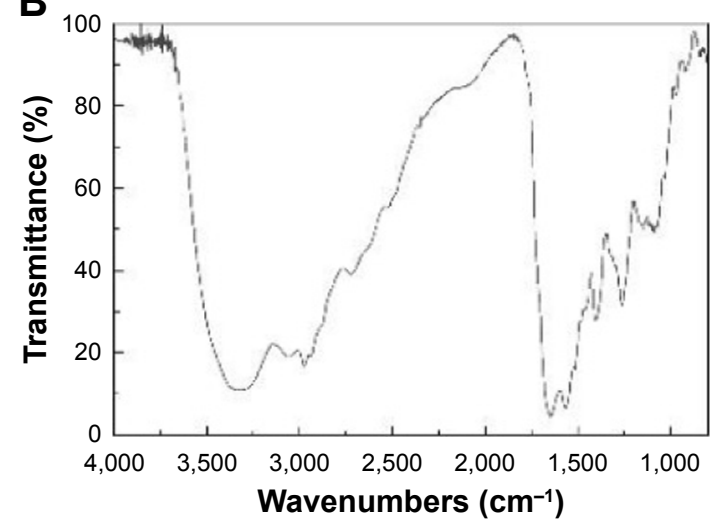

D

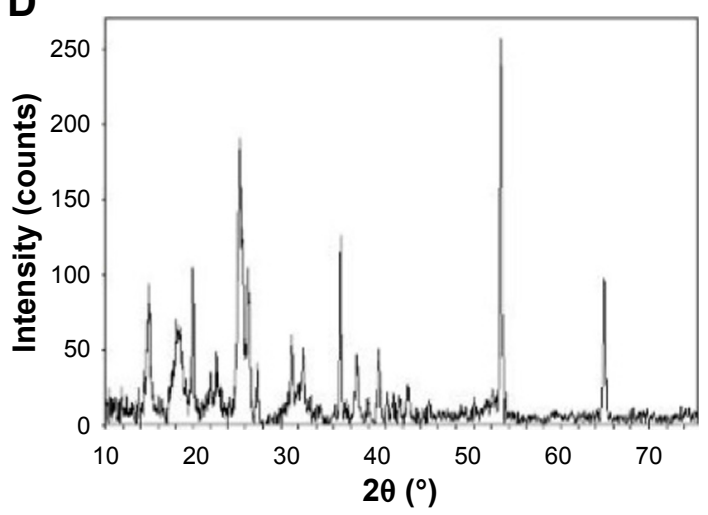

E

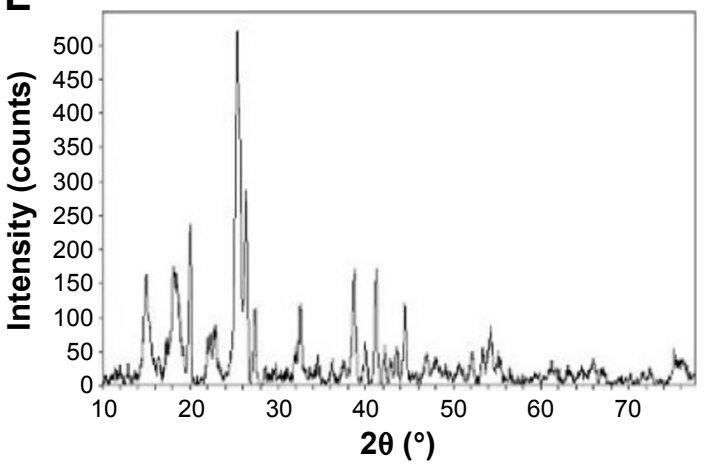

Figure 3 FTIR spectra of the (A) nHA/PA66, (B) SF-nHA/PA66, (C) CNT/SF-nHA/PA66 scaffolds. XRD patterns of the (D) nHA/PA66, and (E) CNT/SFnHA/PA66 scaffolds.

Abbreviations: CNT, carbon nanotube; FTIR, Fourier transform infrared spectra; nHA, nano-hydroxyapatite; PA66, polyamide 66; SF, silk fibroin; XRD, X-ray diffraction.

absorption peaks at 562, 602, 962, and $1,031 \mathrm{~cm}^{-1}$. Figure 3B

shows amide I, II, and III peaks at 1,651, 1,567, and $1,265 \mathrm{~cm}^{-1}$, respectively, demonstrating the surface modification of SF. Figure 3C shows CNT peaks at 3,358, 3,075, 2,975 , and $2,728 \mathrm{~cm}^{-1}$.

Figure 3D shows the X-ray diffraction $2 \theta$ peaks of nHA/ PA66 at $25.9^{\circ}, 31.8^{\circ}, 33.2^{\circ}, 37.5^{\circ}, 47.5^{\circ}, 49.5^{\circ}$, and $55.5^{\circ}$. Figure $3 \mathrm{E}$ indicates that the $\mathrm{CNT} / \mathrm{SF}-\mathrm{nHA} / \mathrm{PA} 66$ scaffold did not exhibit any obvious changes in X-ray diffraction peak positions, but the peak intensity at $25.9^{\circ}$ increased, while the peak intensity at $55.5^{\circ}$ decreased.

\section{Scaffold property}

Table 1 shows the porosity, water binding, and compressive strength of the nHA/PA66 and CNT/SF-nHA/PA66 scaffolds. The porosities of the nHA/PA66 and CNT/SF-nHA/ PA66 scaffolds were $62.22 \%$ and $62.07 \%$, respectively, indicating no obvious changes after $\mathrm{CNT} / \mathrm{SF}$ modification. The compressive strength of CNT/SF-nHA/PA66 was $5.4 \mathrm{MPa}$, representing a $2 \%$ increase over that of the nHA/PA66 scaffold. The water-absorbing rate of the scaffolds changed from $62.51 \%$ to $49.11 \%$ after CNT/SF modification. 
Table I The porosity, water absorption, and mechanical property of CNT/SF-nHA/PA66 scaffolds

\begin{tabular}{lll}
\hline Group & nHA/PA66 & CNT/SF-nHA/PA66 \\
\hline Porosity (\%) & 62.22 & 62.07 \\
Water binding (\%) & $62.5 \mathrm{I} \pm 2.02$ & $49.1 \mathrm{I} \pm 0.68$ \\
Compressive strength (MPa) & 5.3 & 5.4 \\
\hline
\end{tabular}

Note: The results of water absorption ratio obtained from three data values are presented as mean \pm standard deviation, $\mathrm{n}=3$.

Abbreviations: CNT, carbon nanotube; nHA, nano-hydroxyapatite; PA66, polyamide 66; SF, silk fibroin.

The conductivity of the CNT/SF-nHA/PA66 scaffolds is shown in Figure S1. The conductivity increased with the addition of CNT/SF to the scaffold, from 0.001575 $\mathrm{S} / \mathrm{m}$ for the nHA/PA66 scaffold to $0.00336 \mathrm{~S} / \mathrm{m}$ for the $0.2 \mathrm{mg} / \mathrm{mL}$ CNT/SF-nHA/PA66 scaffold. The increase in conductivity by $113 \%$ can be due to the CNTs that are dispersed in the structure of the conductive scaffolds. In addition, the conductivity of the $0.2 \mathrm{mg} / \mathrm{mL} \mathrm{CNT/SF-nHA/}$ PA66 scaffold is close to that of spongy bone in human $(0.01-0.06 \mathrm{~S} / \mathrm{m}),{ }^{28}$ demonstrating the feasibility of applying electrical signals on these scaffolds to accelerate tissue regeneration.

\section{Degradation test}

The weight changes of the CNT/SF-nHA/PA66 scaffold in SBF were determined at different time points to reflect the changes in the rate of degradation. A bone scaffold will form an apatite-like material in the bone marrow after it is implanted in the human body, and the layered structure can increase the compatibility with body tissue. ${ }^{25} \mathrm{SBF}$ is currently the most commonly used simulation solution to test the in vitro biological activity of materials. Figure 4 shows the degradation rate of the CNT/SF-nHA/PA66 scaffold up to 30 days. In the first 12 days, the weight of the scaffold

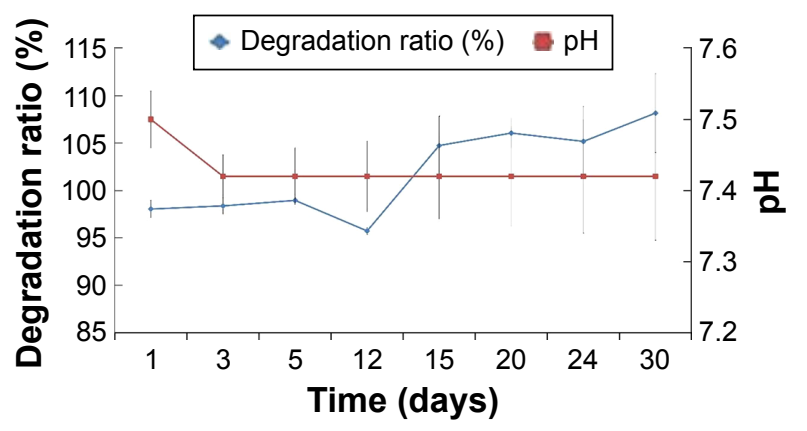

Figure 4 Degradation ratio of CNT/SF-nHA/PA66 scaffolds.

Notes: The degradation samples were evaluated in SBF from I to 30 days. The results obtained from three data values are presented as mean \pm standard deviation, $\mathrm{n}=3$.

Abbreviations: CNT, carbon nanotube; nHA, nano-hydroxyapatite; PA66, polyamide 66; SBF, simulated body fluid; SF, silk fibroin. decreased, indicating the degradation of SF on the surface of the CNT/SF-nHA/PA66 scaffold. In the next 18 days, the weight of the scaffold increased, indicating the deposition of calcium, phosphorus, and other mineral elements on the surface of CNT/SF-nHA/PA66. The $\mathrm{pH}$ of degradation liquid changed over 30 days. At 3 days, the $\mathrm{pH}$ value dropped and then increased. The drop was presumably because of the degradation of acidic amino acids in SF, and the increase was caused by the effect of $\mathrm{CO}_{2}$ in air. The details of highperformance liquid chromatography could be found in Figures S2 and S3 and in Tables S1-S3.

\section{Formulation selection}

A live/dead viability cell image kit was used to evaluate the viability of BMSCs on the scaffolds. The green and red florescence signals correspond to the BMSCs and scaffold, respectively. The confocal images show that BMSCs grew well on the scaffolds, and a higher cell density on the surface of the scaffolds can be observed in Figure 5 and Videos S1, $\underline{\text { S2 }}, \underline{\mathrm{S} 3}$, $\underline{\mathrm{S}}$, and $\underline{\mathrm{S}}$. The images were analyzed using MetaMorph software, and the cell viability percentage values per unit volume on the nHA/PA66, SF-nHA/PA66, $0.2 \mathrm{mg} / \mathrm{mL}$ CNT/SF-nHA/PA66, $0.5 \mathrm{mg} / \mathrm{mL}$ CNT/SF-nHA/PA66, and $1 \mathrm{mg} / \mathrm{mL}$ CNT/SF-nHA/PA66 scaffolds were $24.84 \%$, $16.14 \%, 27.54 \%, 13.70 \%$, and $10.77 \%$, respectively. The $0.2 \mathrm{mg} / \mathrm{mL}$ CNT/SF-nHA/PA66 scaffold showed the best biological compatibility with BMSCs among those tested. Thus, we selected this scaffold for the following study.

\section{Cell attachment}

We used SEM to investigate the cell morphology after culturing on different scaffolds. Figure 6 shows that BMSCs cultured on the nHA/PA66, SF-nHA/PA66, and CNT/SF-nHA/ PA66 scaffolds adhered and grew well; some cells grew into the large hole of the scaffold. The SEM images illustrate that the nHA/PA66 scaffold with surface modification has good cell compatibility.

\section{Drug release behavior}

It is widely known that CNTs have high specific surface area and can easily adsorb DEX. Oxidized CNTs with holes in their pipe ends and sidewalls can adsorb higher amounts of DEX than nontreated CNTs. CNTs can adsorb $200 \mathrm{mg} / \mathrm{g}$ of DEX. In 2005, Murakami et al successfully completed a preliminary experiment on the use of CNTs as a drug delivery system. ${ }^{29}$ DEX-loaded CNTs have been reported to have a good basis and feasibility for use in drug delivery. ${ }^{30-34}$ Oxidized CNTs with holes in their pipe ends and sidewalls 

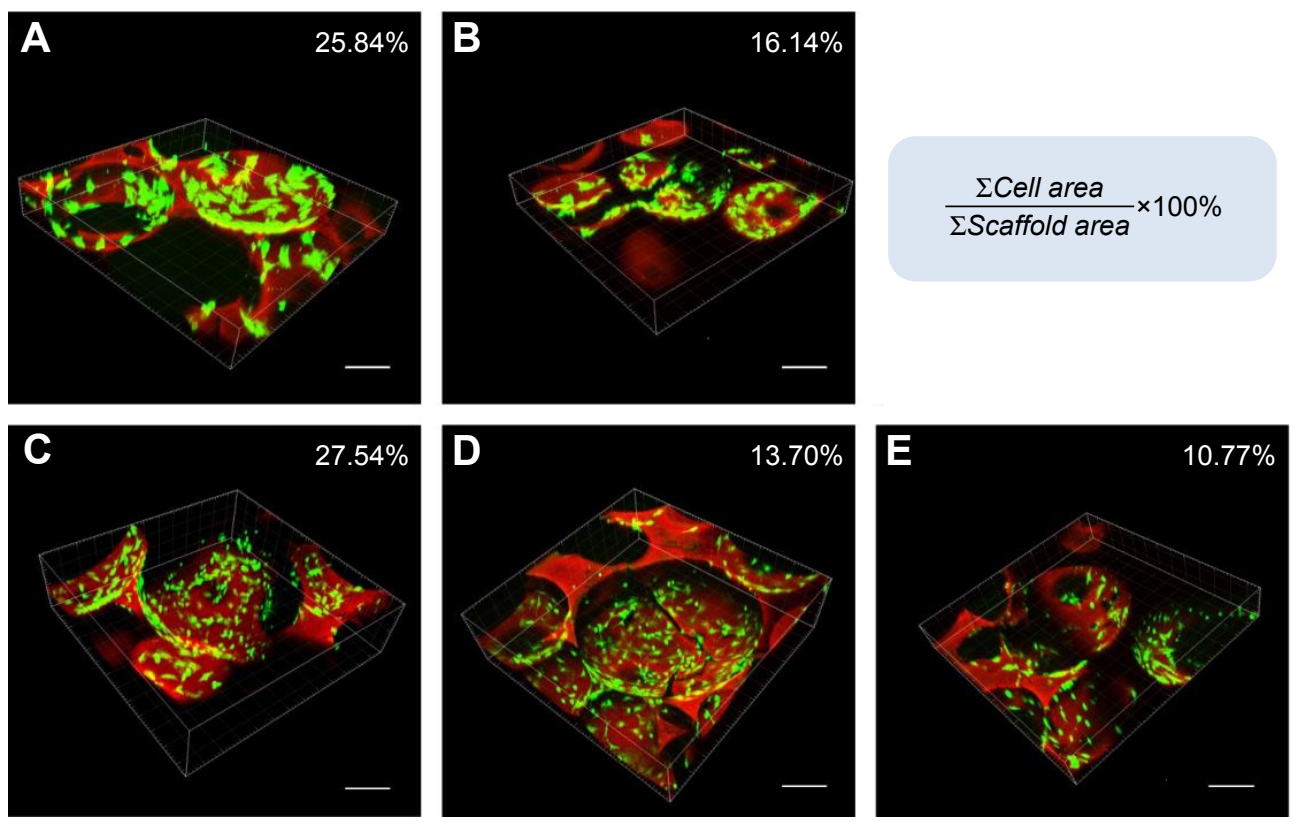

Figure 5 Live/dead cell staining. Confocal images of BMSCs in (A) nHA/PA66, (B) SF-nHA/PA66, (C) 0.2 mg/mL CNT/SF-nHA/PA66, (D) 0.5 mg/mL CNT/SF-nHA/PA66, and $(\mathbf{E}) \mathrm{I} \mathrm{mg} / \mathrm{mL} \mathrm{CNT} / \mathrm{SF}-\mathrm{nHA} / \mathrm{PA} 66$ scaffolds.

Notes: Green fluorescence: BMSCs; red fluorescence: scaffold. Bar $=200 \mu \mathrm{m}$. MetaMorph software was used to analyze the amount of red fluorescence ( $\Sigma$ Scaffold area) and green fluorescence $\left(\Sigma C\right.$ ell area). The cell viability of BMSCs on the scaffold was calculated using the following equation: cell viability $\%=\left(\sum\right.$ Cell area/ $\Sigma$ Scaffold area $) \times 100 \%$. Abbreviations: BMSCs, bone mesenchymal stem cells; CNT, carbon nanotube; nHA, nano-hydroxyapatite; PA66, polyamide 66; SF, silk fibroin.

can adsorb higher amounts of DEX by $\pi-\pi$ interactions of aromatic moieties. ${ }^{35}$

Figure 7 shows the cumulative amount of DEX released from drug-loaded CNT/SF-nHA/PA66 scaffolds. DEX was released at levels of $32 \%$ and $28 \%$ in $0.5 \%$ SDS and PBS, respectively, at 2 hours. At 8 hours, DEX was released at a level of $60 \%$ in $0.5 \%$ SDS and $57 \%$ in PBS. Within 24 hours, the cumulative amount of DEX released was $97 \%$ in $0.5 \%$ SDS and $66 \%$ in PBS. At 6 days, the cumulative amount of DEX released reached $98 \%$ in $0.5 \%$ SDS and $73 \%$ in PBS. It was speculated that DEX was released mainly through diffusion during the first
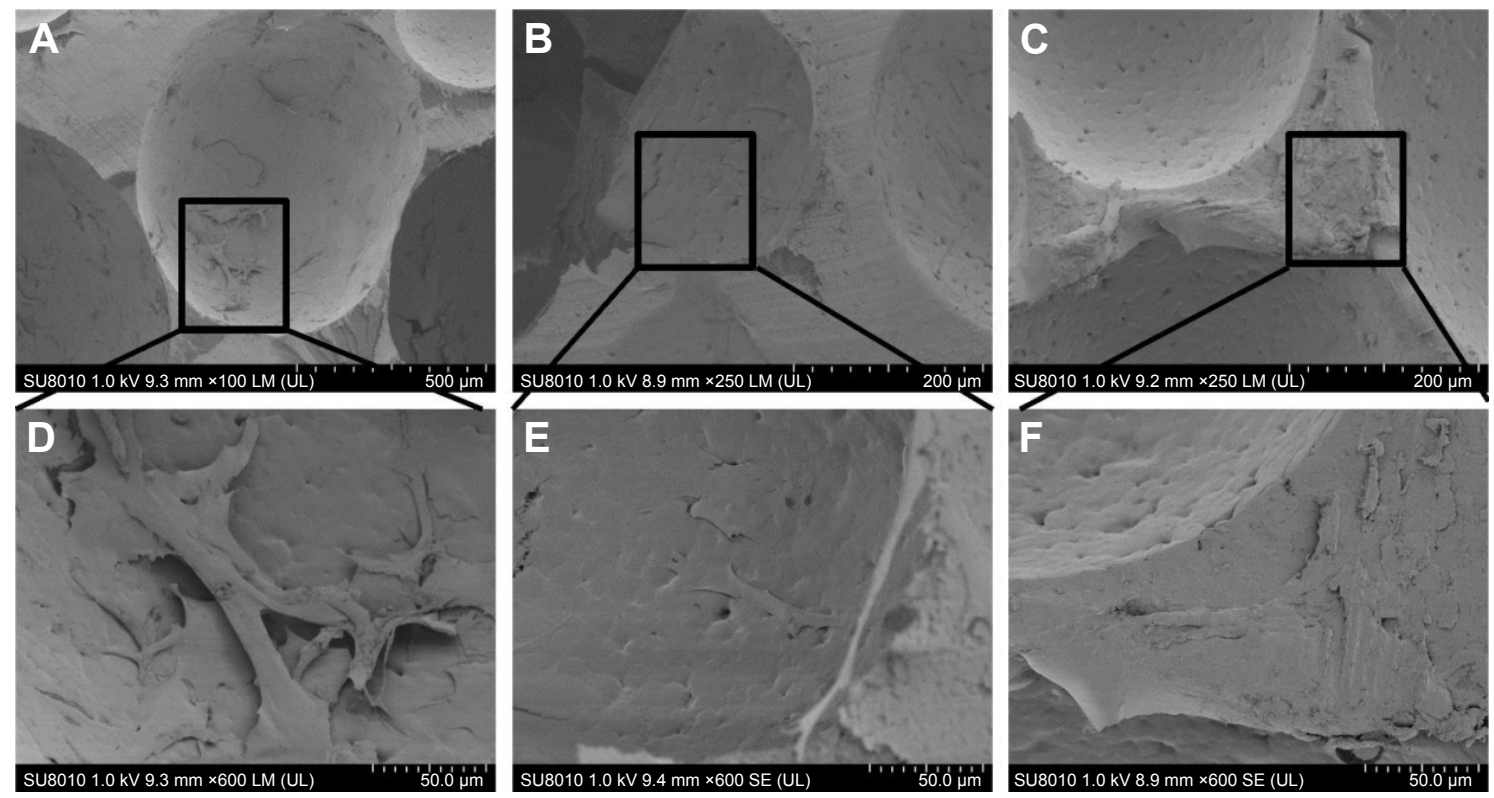

Figure 6 SEM photographs of the BMSCs cultured on (A, D) nHA/PA66, (B, E) SF-nHA/PA66, and (C, F) $0.2 \mathrm{mg} / \mathrm{mL}$ CNT/SF-nHA/PA66 scaffolds for 7 days.

Abbreviations: BMSCs, bone mesenchymal stem cells; CNT, carbon nanotube; nHA, nano-hydroxyapatite; PA66, polyamide 66; SEM, scanning electron microscopy; SF, silk fibroin. 


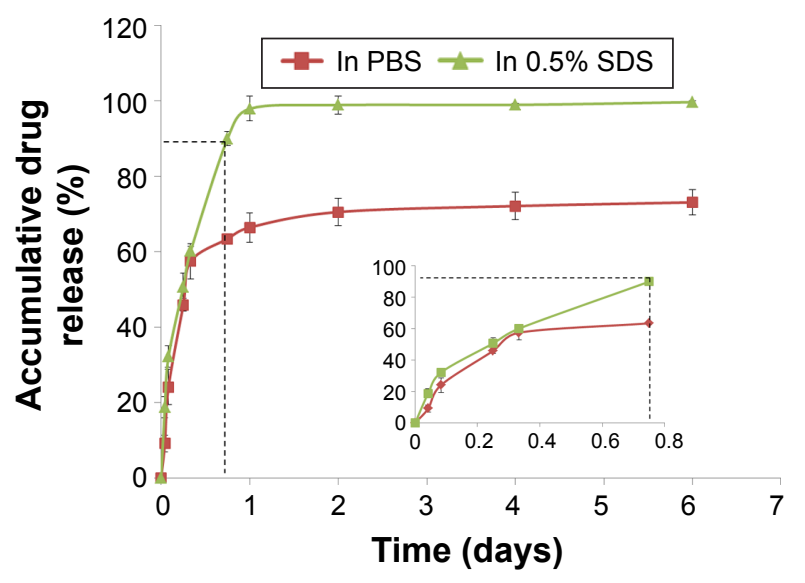

Figure 7 Drug release profiles in different release media.

Notes: The cumulative amount of DEX released from DEX-loaded CNT/SF-nHA/ PA66 scaffolds in dialysis bags $(3,000 \mathrm{MW})$ contained a release medium consisting of either PBS ( $\mathrm{pH} 7.4$ ) or $0.5 \%$ SDS in PBS. Inset shows magnified area identified by the dotted lines. The results obtained from three data values are presented as mean \pm standard deviation, $\mathrm{n}=3$.

Abbreviations: $\mathrm{CNT}$, carbon nanotube; $\mathrm{DEX}$, dexamethasone; $\mathrm{nHA}$, nanohydroxyapatite; PA66, polyamide 66; PBS, phosphate-buffered saline; SDS, sodium dodecyl sulfate; SF, silk fibroin.

24 hours, and further drug release occurred slowly after 24 hours.

\section{ALP activity assay}

As shown in Figure 8, the DEX-loaded CNT/SF-nHA/PA66 scaffold had an inducing effect on BMSC osteogenesis. Different concentrations of DEX had different effects on BMSC osteogenesis. At a DEX concentration of $1 \mathrm{mg} / \mathrm{mL}$, the DEX-loaded CNT/SF-nHA/PA66 scaffold had the strongest effect on promoting the secretion of ALP.

\section{Discussion}

The healing of bone defects has always been a challenge. Traditional treatments include autogenous bone graft, allograft bone transplantation, and artificial bone material transplantation. However, the source of autologous bone grafts is limited and the surgery causes trauma to the patient. Allograft bone transplantation may induce immunological rejection, and the osteogenesis performance of artificial bone transplantation material is uncertain. Bone tissue engineering has opened up a new path for the clinical treatment of bone defects. Composite scaffolds with a biomimetic structure and good biological compatibility, osteoconductivity, and osteoinductivity are currently a key point for bone tissue engineering. ${ }^{36,37}$ Some inorganic materials have been reported to improve the mechanical and biological properties of bone tissue scaffold. ${ }^{38,39}$

The nHA/PA66 porous scaffolds fabricated by a phase inversion method provide a good imitation of natural bone and have been widely used in orthopedics. Xu et a ${ }^{40}$ investigated a porous nHA/PA66 scaffold material and showed that three-dimensional pore structures could facilitate cell adhesion, differentiation, and proliferation, and promote fibrovascular and nerve colonization. Porous n-HA/PA66 scaffold material could be a good candidate as bone substitute material because of its excellent histocompatibility, osteoconductivity, and osteoinductivity. Multiphase composite scaffolds or surface-modified scaffolds based on nHA/PA66 two-phase composites have been fabricated in order to build scaffolds that are more biocompatible.

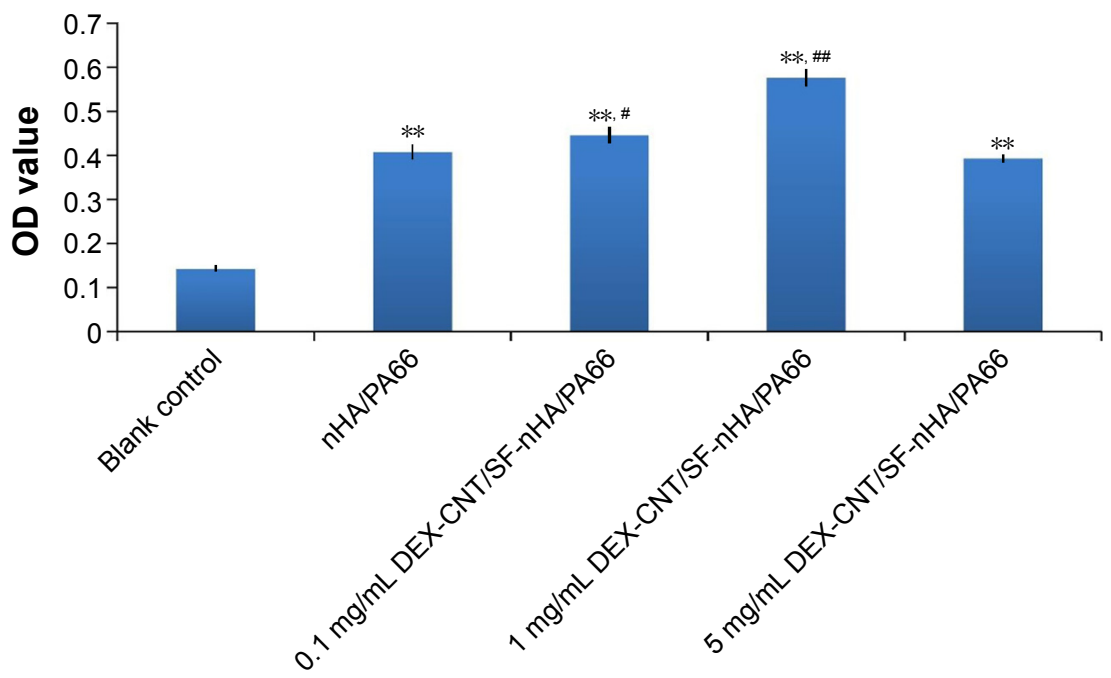

Figure 8 ALP activity of the BMSCs cultured on drug-loaded CNT/SF-nHA/PA66 scaffolds for 14 days.

Notes: The different drug-loaded CNT/SF-nHA/PA66 scaffolds with various DEX concentrations ( 0.1 , I, and $5 \mathrm{mg} / \mathrm{mL})$ and the $\mathrm{nHA} / \mathrm{PA} 66 \mathrm{scaffolds}$ were evaluated regarding the ALP activity of BMSCs on the scaffold for I 4 days. Data are presented as mean \pm standard deviation, $n=5$; $* * P<0.01$ vs blank control, ${ }^{\# P<0.05}$, and ${ }^{\#} P<0.0$ I vs $n H A /$ PA66 scaffold.

Abbreviations: ALP, alkaline phosphatase; BMSCs, bone mesenchymal stem cells; CNT, carbon nanotube; DEX, dexamethasone; nHA, nano-hydroxyapatite; OD, optical density; PA66, polyamide 66; SF, silk fibroin. 
With further development of bone tissue engineering, the use of drug-eluting stents has become a popular trend. ${ }^{41}$ Drug-eluting stents loaded with growth factors or antiinflammatory drugs can promote bone tissue repair or treat an infection. ${ }^{42-45}$ Our research is the first to fabricate the drugloaded nHA/PA66 conjugated CNTs scaffold. The CNTs improved the mechanical and conductive properties of nHA/ PA66 scaffold. Also, the DEX-loaded CNT/SF-nHA/PA66 composite scaffold could successfully promote osteogenic differentiation of BMSCs. This system opened up a brand new way for bone tissue engineering.

In our study, a porous nHA/PA66 scaffold was modified with MWCNT-COOH and SF by freeze-drying and chemical cross-linking methods in order to further improve the growth environment of the bone interface after implant. We used CNTs at a concentration of $0.2 \mathrm{mg} / \mathrm{mL}$ in a CNT/ SF mixture to modify the nHA/PA66 scaffolds and loaded DEX into CNT to fabricate a drug-loaded CNT/SF-nHA/ PA66 scaffold that could promote the osteogenic differentiation of BMSCs.

The topological structure as well as aperture of scaffolds is extremely important for cell growth and nutrient transport. The SEM results presented in Figure 3 show that the average diameters of the CNT/SF-nHA/PA66 scaffolds were $\sim 500 \mu \mathrm{m}$, meeting the requirement of a bone tissue scaffold. ${ }^{27}$ The freeze-drying combined with chemical crosslinking method successfully modified the surface of nHA/ PA66 and did not destroy the original pore structure of the scaffold. In addition, the porosity and the pore diameter of a bone tissue engineering scaffold are extremely important for cell growth, nutrient transport, and bone formation. High values of porosity and specific surface area enhance the adhesion and growth of seed cells, deposition of extracellular matrix, and delivery of nutrients and oxygen. ${ }^{46}$ The porosity of the CNT/SF-nHA/PA66 scaffold is about $62 \%$, meeting the requirement for bone tissue engineering. Moreover, the hydrophobicity of a bone scaffold can affect its compatibility. A high water absorption value is not conducive to seed-cell adhesion, proliferation, and osteogenesis, and thus affects the ingrowth of new bone tissue. ${ }^{47}$ The water absorption value of a polymer is related to the polarity of the chemical bonds among the molecules. The polarity of the amide bonds in PA66 is very strong; thus, it can easily form hydrogen bonds with water molecules, which leads to a high water absorption value. Since CNTs and SF have hydrophobic structures, the water absorption of the CNT/SF-nHA/PA66 scaffold is lower than that of the nHA/PA66 scaffold. The demand for bone tissue engineering scaffolds is relatively high, and they must possess not only a porous structure and good biocompatibility but also sufficient mechanical strength and bone conductibility. The necessity for both a porous structure and mechanical strength can be difficult to achieve because as porosity increases, the mechanical strength decreases. The challenge in bone scaffold preparation is to improve the mechanical strength while ensuring a porous structure. The results presented in Table 1 show that the compressive strength increased by $2 \%$ after surface modification with CNTs and SF. The results presented in Figure 4 show the form of hydroxyapatite developed on the surface of the CNT/ SF-nHA/PA66 scaffold in the final 15 days, demonstrating its good mineralization ability in vitro.

Cell adhesion is an important reference value to measure the compatibility of scaffold materials. The selection of the surface features of a material is an important factor because cells respond to microscale or nanoscale surface structures, and contact with the surface regulates cell adhesion and growth behavior. ${ }^{48}$ Currently, one problem in the bone tissue engineering field is insufficient cell adhesion, which affects cell proliferation, differentiation, and osteogenesis. ${ }^{49}$ The cell experiment shown in Figure 5 indicates that BMSCs cultured on the CNT/SF-nHA/PA66 scaffold exhibited good adhesion and proliferation abilities.

DEX can promote the expression of osteoblast genes and is a commonly used small-molecule drug for inducing the osteogenic differentiation of BMSCs. ${ }^{50}$ The expression of ALP in the extracellular matrix is considered to be an early sign of BMSC maturation, reflecting differentiation into mature bone cells and matrix mineralization. ${ }^{51}$ Figure 6 shows that the drug-loaded scaffold had an osteogenesis-inducing effect on BMSCs, and different concentrations of DEX had different effects on BMSCs. At a concentration of $1 \mathrm{mg} / \mathrm{mL}$, DEX had the strongest effect on increasing ALP activity. The drug release behavior of DEX can promote the osteogenic differentiation of BMSCs. All these results indicate that the CNT/SF-nHA/PA66 scaffold fulfills the basic requirements of bone tissue engineering, and it has potential for application in bone defect repair.

\section{Conclusion}

The results of our study showed that the nHA/PA66 scaffolds modified with CNTs and SF by freeze-drying and cross-linking are useful in bone tissue engineering. The DEX-loaded CNT/SF-nHA/PA66 composite scaffold can promote osteogenic differentiation of BMSCs, and the drug-loaded scaffolds are expected to be effective as bone tissue engineering scaffolds. 


\section{Acknowledgments}

We are grateful to the Core Facility of Zhejiang University of Medicine for technical assistance in confocal laser scanning microscopy. Also, we would like to acknowledge the Analysis Center of Agrobiology and Environmental Science for assistance in transmission electron microscopy service.

\section{Disclosure}

The authors report no conflicts of interest in this work.

\section{References}

1. Silvestris F, Lombardi L, De Matteo M, Bruno A, Dammacco F. Myeloma bone disease: Pathogenetic mechanisms and clinical assessment. Leuk Res. 2007;31(2):129-138.

2. Cypher TJ, Grossman JP. Biological principles of bone graft healing. J Foot Ankle Surg. 1996;35(5):413-417.

3. Crane GM, Ishaug SL, Mikos AG. Bone tissue engineering. Nat Med. 1995;1(12):1322-1324.

4. Black CR, Goriainov V, Gibbs D, Kanczler J, Tare RS, Oreffo RO Bone tissue engineering. Curr Mol Biol Rep. 2015;1(3):132-140.

5. Mistry AS, Mikos AG. Tissue engineering strategies for bone regeneration. Adv Biochem Eng Biotechnol. 2005;94:1-22.

6. Gong T, Xie J, Liao J, Zhang T, Lin S, Lin Y. Nanomaterials and bone regeneration. Bone Res. 2015;3:15029.

7. Burg KJL, Porter S, Kellam JF. Biomaterial developments for bone tissue engineering. Biomaterials. 2000;21(23):2347-2359.

8. Agarwal R, Garcia AJ. Biomaterial strategies for engineering implants for enhanced osseointegration and bone repair. Adv Drug Deliv Rev. 2015;94:53-62.

9. Peter SJ, Miller MJ, Yasko AW, Yaszemski MJ, Mikos AG. Polymer concepts in tissue engineering. J Biomed Mater Res. 1998;43(4): 422-427.

10. Liu X, Ma PX. Polymeric scaffolds for bone tissue engineering. Ann Biomed Eng. 2004;32(3):477-486.

11. Jahan K, Tabrizian M. Composite biopolymers for bone regeneration enhancement in bony defects. Biomater Sci. 2016;4(1):25-39.

12. Ye L, Su Q, Zhou X-D. Biological effects of nano-hydroxyapatite/ polyamide 66 on the osteoblast. Hua Xi Kou Qiang Yi Xue Za Zhi. 2005;23(2):142-144.

13. Wang X, Li Y, Wei J, de Groot K. Development of biomimetic nano-hydroxyapatite/poly(hexamethylene adipamide) composites. Biomaterials. 2002;23(24):4787-4791.

14. Min PL, Dian MJ, Zheng XQ. Experimental research on in vitro antibacterial property and biocompatibility of bone filling materials of $\mathrm{TiO}_{2}$-Ag-nHA/PA66. Orthop J China. 2010;18(6):493-497.

15. Fan J, Chang S, Dong M, Huang D, Li J, Jiang D. Study on the development of Ag-nano-hydroxyapatite/polyamide 66 porous scaffolds with surface mineralization. J Biomed Eng. 2012;29(6):1119-1124.

16. Hutmacher DW, Schantz JT, Lam CXF, Tan KC, Lim TC. State of the art and future directions of scaffold-based bone engineering from a biomaterials perspective. J Tissue Eng Regen M. 2007;1(4): 245-260.

17. Ferreira L, Karp JM, Nobre L, Langer R. New opportunities: the use of nanotechnologies to manipulate and track stem cells. Cell Stem Cell. 2008;3(2):136-146.

18. Bari S, Chu PPY, Lim A, et al. Protective role of functionalized single walled carbon nanotubes enhance ex vivo expansion of hematopoietic stem and progenitor cells in human umbilical cord blood. Nanomedicine. 2013;9(8):1304-1316.

19. Zhao B, Hu H, Mandal SK, Haddon RC. A bone mimic based on the self-assembly of hydroxyapatite on chemically functionalized singlewalled carbon nanotubes. Chem Mater. 2005;17(12):3235-3241.
20. Mandal BB, Grinberg A, Gil ES, Panilaitis B, Kaplan DL. High-strength silk protein scaffolds for bone repair. Proc Natl Acad Sci US A. 2012; 109(20):7699-7704.

21. Wenk E, Wandrey AJ, Merkle HP, Meinel L. Silk fibroin spheres as a platform for controlled drug delivery. J Control Release. 2008; 132(1):26-34.

22. Kong XD, Cui FZ, Wang XM, Zhang M, Zhang W. Silk fibroin regulated mineralization of hydroxyapatite nanocrystals. J Cryst Growth. 2004;270(1-2):197-202.

23. Koh LD, Cheng Y, Teng CP, et al. Structures, mechanical properties and applications of silk fibroin materials. Prog Polym Sci. 2015; 46:86-110.

24. Pan Y-X, Yu Z-Z, Ou Y-C, Hu G-H. A new process of fabricating electrically conducting nylon 6/graphite nanocomposites via intercalation polymerization. J Polym Sci Part B Polym Phys. 2000;38(12): 1626-1633.

25. Kokubo T, Takadama H. How useful is SBF in predicting in vivo bone bioactivity? Biomaterials. 2006;27(15):2907-2915.

26. Li XY, Zhang Y, Qi GX. Evaluation of isolation methods and culture conditions for rat bone marrow mesenchymal stem cells. Cytotechnology. 2013;65(3):323-334.

27. Bohner M, Loosli Y, Baroud G, Lacroix D. Commentary: deciphering the link between architecture and biological response of a bone graft substitute. Acta Biomater. 2011;7(2):478-484.

28. Gabriel S, Lau RW, Gabriel C. The dielectric properties of biological tissues: II. Measurements in the frequency range $10 \mathrm{~Hz}$ to $20 \mathrm{GHz}$. Phys Med Biol. 1996;41(11):2251.

29. Murakami T, Ajima K, Miyawaki J, Yudasaka M, Iijima S, Shiba K. Drug-loaded carbon nanohorns: adsorption and release of dexamethasone in vitro. Mol Pharm. 2004;1(6):399-405.

30. Lodhi N, Mehra NK, Jain NK. Development and characterization of dexamethasone mesylate anchored on multi walled carbon nanotubes. J Drug Target. 2013;21(1):67-76.

31. Wei W-L, Chen Q, Li H, Lin J-M. Self-assemblies of single-walled carbon nanotubes through tunable tethering of pyrenes by dextrin for rapidly chiral sensing. Int J Anal Chem. 2011;2011:862692.

32. Naficy S, Razal JM, Spinks GM, Wallace GG. Modulated release of dexamethasone from chitosan-carbon nanotube films. Sensor Actuat A-Phys. 2009;155(1):120-124.

33. Kim J-H, Heller DA, Jin $\mathrm{H}$, et al. The rational design of nitric oxide selectivity in single-walled carbon nanotube near-infrared fluorescence sensors for biological detection. Nat Chem. 2009;1(6):473-481.

34. Kitano H, Tachimoto $\mathrm{K}$, Gemmei-Ide $\mathrm{M}$, Tsubaki N. Interaction between polymer chains covalently fixed to single-walled carbon nanotubes. Macromol Chem Phys. 2006;207(9):812-819.

35. Klumpp C, Kostarelos K, Prato M, Bianco A. Functionalized carbon nanotubes as emerging nanovectors for the delivery of therapeutics. Biochim Biophys Acta. 2006;1758(3):404-412.

36. Costa MA, Simon DI. Molecular basis of restenosis and drug-eluting stents. Circulation. 2005;111(17):2257-2273.

37. Garg T, Singh O, Arora S, Murthy R. Scaffold: a novel carrier for cell and drug delivery. Crit Rev Ther Drug Carrier Syst. 2012;29(1):1-63.

38. Feng P, Guo X, Gao C, et al. Diopside modified porous polyglycolide scaffolds with improved properties. RSC Adv. 2015;5(68): 54822-54829.

39. Feng P, Wei P, Shuai C, Peng S. Characterization of mechanical and biological properties of 3-D scaffolds reinforced with zinc oxide for bone tissue engineering. PLoS One. 2014;9(1):e87755.

40. Xu Q, Lu H, Zhang J, Lu G, Deng Z, Mo A. Tissue engineering scaffold material of porous nanohydroxyapatite/polyamide 66. Int $J$ Nanomedicine. 2010;5:331-335.

41. Porter JR, Ruckh TT, Popat KC. Bone tissue engineering: a review in bone biomimetics and drug delivery strategies. Biotechnol Progr. 2009; 25(6):1539-1560.

42. Xiang B, Guo Y. Fabrication of drug-loaded tissue engineering scaffold and its curative effect for chronic osteomyelitis in rabbits. Chin J Traumatol. 2015;31(3):212-216. 
43. Zhang X, Zhang J, Shi B. Mesoporous bioglass/silk fibroin scaffolds as a drug delivery system: Fabrication, drug loading and release in vitro and repair calvarial defects in vivo. J Wuhan Univ Technol. 2014;29(2): 401-406.

44. Liu H, Zhang L, Shi P, Zou Q, Zuo Y, Li Y. Hydroxyapatite/polyurethane scaffold incorporated with drug-loaded ethyl cellulose microspheres for bone regeneration. J Biomed Mater Res B Appl Biomater. 2010; 95B(1):36-46.

45. Liu H-H, Zhang J-H, Xu Q-L, Zhang L, Li Y-B. Studies on hydroxyapatite/ polyurethane scaffold containing drug-loaded microspheres for bone tissue engineering. J Inorg Mater. 2011;26(10):1073-1077.

46. Karageorgiou V, Kaplan D. Porosity of 3D biomaterial scaffolds and osteogenesis. Biomaterials. 2005;26(27):5474-5491.

47. Yang CY, Huang LY, Shen TL, Yeh JA. Cell adhesion, morphology and biochemistry on nano-topographic oxidized silicon surfaces. Eur Cells Mater. 2010;20:415-430.
48. Garcia AJ, Reyes CD. Bio-adhesive surfaces to promote osteoblast differentiation and bone formation. J Dent Res. 2005;84(5):407-413.

49. Shekaran A, Garcia AJ. Extracellular matrix-mimetic adhesive biomaterials for bone repair. J Biomed Mater Res A. 2011;96(1): 261-272.

50. Zhou H, Mak W, Zheng Y, Dunstan CR, Seibel MJ. Osteoblasts directly control lineage commitment of mesenchymal progenitor cells through Wnt signaling. J Biol Chem. 2008;283(4):1936-1945.

51. Genge BR, Sauer GR, Wu LN, McLean FM, Wuthier RE. Correlation between loss of alkaline phosphatase activity and accumulation of calcium during matrix vesicle-mediated mineralization. J Biol Chem. 1988; 263(34):18513-18519. 


\section{Supplementary materials \\ Method}

\section{BMSC isolation, purification, and culture}

Sprague Dawley rats were euthanized by cervical dislocation and the femora explanted under aseptic conditions. The bone marrow was flushed out into $25 \mathrm{~mL}$ of a modified isolation medium including low-glucose Dulbecco's Modified Eagle's Medium (DMEM), 10\% fetal bovine serum, L-glutamine, penicillin, and streptomycin by using a $20 \mathrm{~mL}$ injector, filtered through a sieve, and then centrifuged at 1,200 rpm for 5 minutes. The pellet was resuspended in $10 \mathrm{~mL}$ of modified DMEM-low glucose medium. The suspension was then incubated in $25 \mathrm{~cm}^{2}$ flasks at $37^{\circ} \mathrm{C}$ in a humidified atmosphere with $5 \% \mathrm{CO}_{2}$. The medium was refreshed after 48 hours of culture and then replaced every other day. Cell passaging was performed when the cell confluence reached $90 \%$. The second-passage cells at subconfluence were used for all experiments up to the fifth passage.

\section{High-performance liquid chromatography}

Conditions for the high-performance liquid chromatograph detection of dexamethasone: chromatographic column, Diamonsil C18 (5 $\mu \mathrm{m}, 250 \times 4.6 \mathrm{~mm}$ ); mobile phase, acetonitrile monohydrate (4:6); flow rate, $1 \mathrm{~mL} / \mathrm{min}$; sample quantity, $20 \mu \mathrm{L}$; detection wavelength, $240 \mathrm{~nm}$; column temperature, $25^{\circ} \mathrm{C}$.

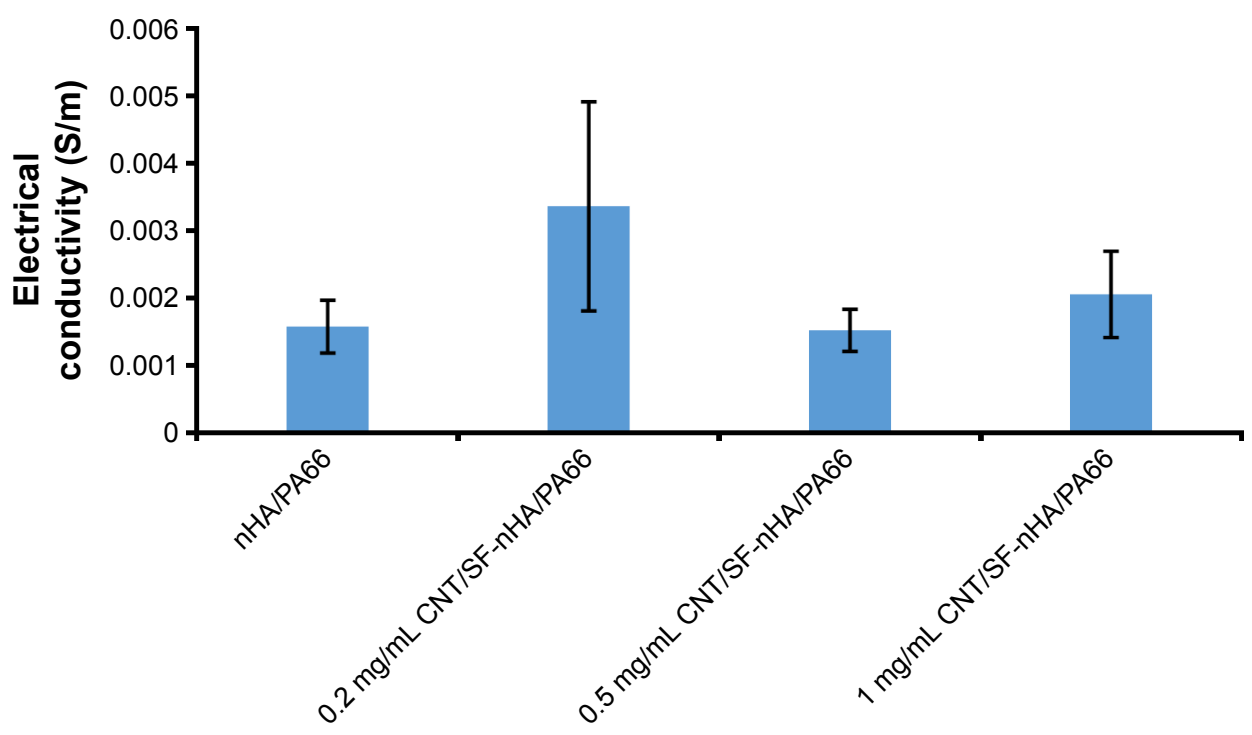

Figure SI The electrical conductivity of nHA/PA66, $0.2 \mathrm{mg} / \mathrm{mL}$ CNT/SF-nHA/PA66, $0.5 \mathrm{mg} / \mathrm{mL}$ CNT/SF-nHA/PA66, and I mg/mL CNT/SF-nHA/PA66 scaffolds. Abbreviations: CNT, carbon nanotube; $\mathrm{nHA}$, nano-hydroxyapatite; PA66, polyamide 66; SF, silk fibroin.

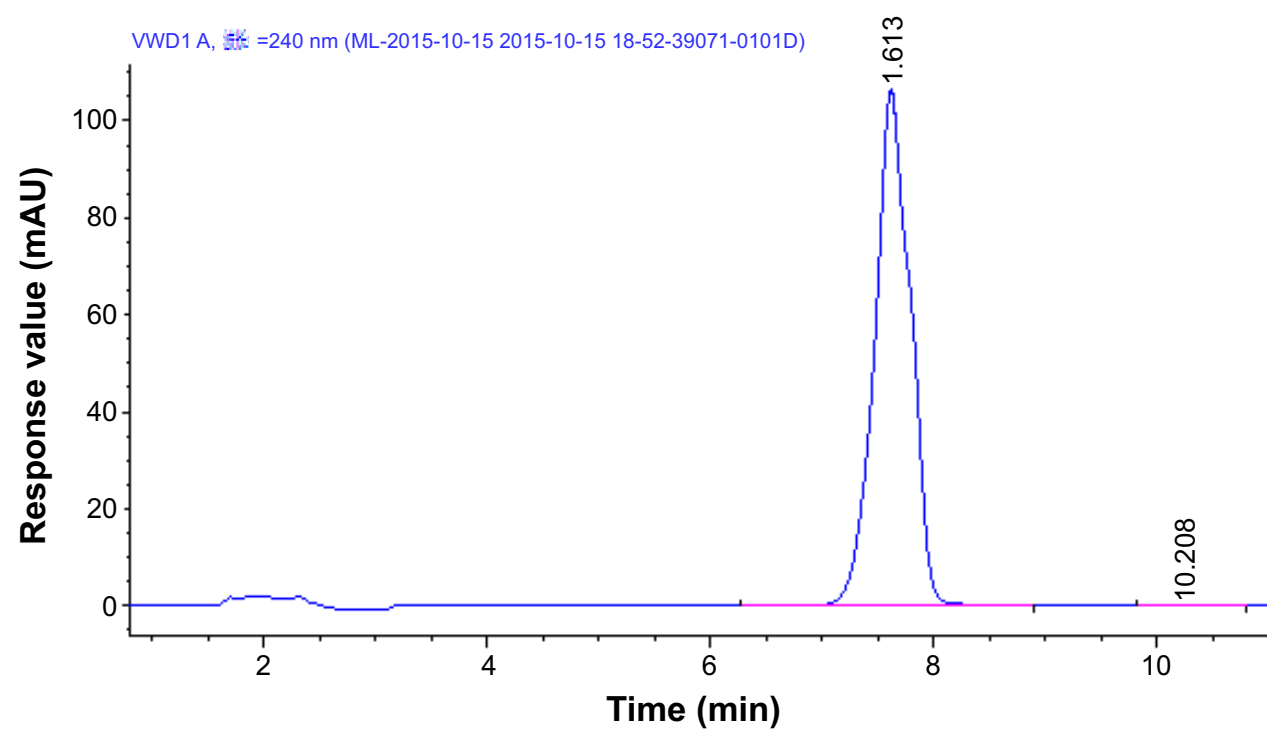

Figure S2 HPLC fingerprint spectrum of DEX.

Notes: Chromatographic column, Diamonsil CI 8 (5 $\mu \mathrm{m}, 250 \times 4.6 \mathrm{~mm})$; mobile phase, acetonitrile monohydrate (4:6); flow rate, I mL/min; sample quantity, $20 \mu \mathrm{L}$; detection wavelength, $240 \mathrm{~nm}$; column temperature, $25^{\circ} \mathrm{C}$.

Abbreviations: DEX, dexamethasone; HPLC, high-performance liquid chromatography. 


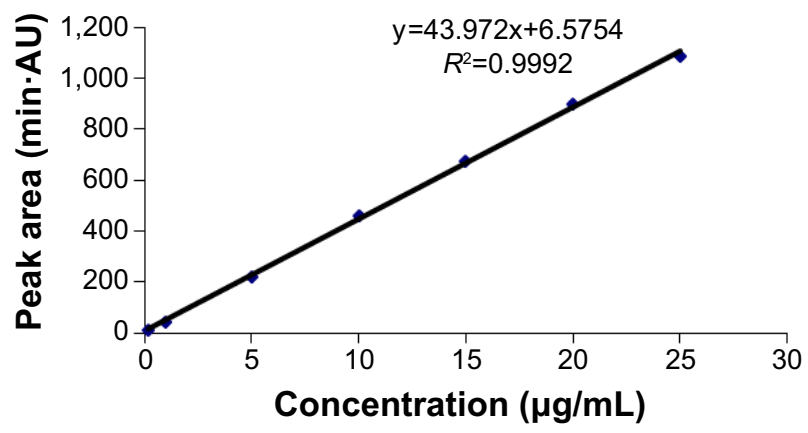

Figure S3 HPLC standard curve of DEX.

Abbreviations: DEX, dexamethasone; HPLC, high-performance liquid chromatography.

Table SI The result of intraday and interday precision $(n=5)$

\begin{tabular}{|c|c|c|c|c|}
\hline \multirow{2}{*}{$\begin{array}{l}\text { Concentration } \\
(\mu \mathrm{g} / \mathrm{mL})\end{array}$} & \multicolumn{2}{|c|}{ Within-day } & \multicolumn{2}{|c|}{ Between-day } \\
\hline & Mean & RSD (\%) & Mean & RSD (\%) \\
\hline 5 & 229.70 & 0.31 & 227.00 & 1.90 \\
\hline 10 & 449.87 & 0.07 & 466.38 & 1.70 \\
\hline 15 & 699.03 & 0.22 & 675.56 & 0.70 \\
\hline
\end{tabular}

Note: Method validation of HPLC.

Abbreviations: HPLC, high-performance liquid chromatography; RSD, relative standard deviation.

Table S2 The stability of dexamethasone $(n=3)$

\begin{tabular}{llll}
\hline Time (h) & Peak & Mean & RSD (\%) \\
\hline 0 & $1,018.9$ & $1,021.8$ & 0.3 \\
4 & $1,020.9$ & \\
8 & $1,027.1$ & & \\
12 & $1,024.2$ & & \\
24 & $1,018.0$ & \\
\hline
\end{tabular}

Note: Method validation of HPLC.

Abbreviations: HPLC, high-performance liquid chromatography; RSD, relative standard deviation.

Table S3 The recovery rate $(n=5)$

\begin{tabular}{lllll}
\hline Added $(\mu \mathrm{g} / \mathrm{mL})$ & Recovered $(\mu \mathrm{g} / \mathrm{mL})$ & Recovery $(\%)$ & Mean $(\%)$ & 95.96 \\
\hline 1.90 & 1.84 & 96.84 & & \\
& 1.82 & 95.79 & & \\
7.60 & 1.81 & 95.26 & 96.54 & 0.16 \\
& 7.33 & 96.45 & & \\
18.24 & 7.35 & 96.71 & 96.87 & 0.23 \\
& 7.33 & 96.54 & & \\
\hline
\end{tabular}

Note: Method validation of HPLC.

Abbreviations: HPLC, high-performance liquid chromatography; RSD, relative standard deviation.

International Journal of Nanomedicine

\section{Publish your work in this journal}

The International Journal of Nanomedicine is an international, peerreviewed journal focusing on the application of nanotechnology in diagnostics, therapeutics, and drug delivery systems throughout the biomedical field. This journal is indexed on PubMed Central, MedLine, CAS, SciSearch ${ }^{\circledR}$, Current Contents $® /$ Clinical Medicine,

\section{Dovepress}

Journal Citation Reports/Science Edition, EMBase, Scopus and the Elsevier Bibliographic databases. The manuscript management system is completely online and includes a very quick and fair peer-review system, which is all easy to use. Visit http://www.dovepress.com/ testimonials.php to read real quotes from published authors. 